\title{
A PROBABLE MODEL OF COMPACT VARIABLE RADIO SOURCES WITH A "SUPERLUMINAL" MOTION OF VLBI-COMPONENTS
}

\author{
B.V. Komberg \\ Space Research Institute \\ Moscow, USSR
}

\begin{abstract}
Ballistic" models are now put in doubt on the strength of the observed properties of "superluminal" components, and preference is given to "screen" models. It is suggested that active nuclei are supermassive systems, such as SS-433, with two precessing relativistic plasma jets, which flow from opposite directions into the same precession cone. In this case "superluminal" components can manifest themselves as displacements of the interaction between these jets and inhomogeneities in the circum-nuclear medium (clouds). Thus, due to isotropic production of relativistic particles in the interaction area and/or magnetic field distortions, bursts of emission may be observed not only along flows, as would be the case for BL Lacertae objects, but also at appreciable angular distances from the jets. Moreover, one may understand the observed one-sidedness of VLBI-jets on parsec scales and the symmetrical VLA-structures on kiloparsec scales as a result of random cloud hits by the precessing flows. We suggest that the observed repetition of active cycles of different objects may be caused by several cloud hits by the precessing flows of a complex multibeam structure once every 50-100 years.
\end{abstract}

Connecting the duration of an emission burst $(\Delta \tau)$ with the time of one beam passing across one cloud, one can estimate its angle size $(\Delta \phi)$ using a period of precession ( $P_{\text {prec }}$ ), a distance from the nucleus to the interaction area $(R)$, and a cone angle $(\alpha)$ :

$$
\Delta \phi=\mathrm{V}_{\text {prec }} \Delta \tau / \mathrm{R} \text {, where } \mathrm{V}_{\text {prec }}=2 \pi \mathrm{R} \operatorname{Sin} \frac{\alpha}{2} \mathrm{P}_{\text {prec }} \cdot
$$

Using $R=10 \mathrm{pc}, \alpha=60^{\circ}, \mathrm{P}_{\text {prec }}=300$ years and $\Delta \tau=3$ years one obtains $V_{\text {prec }}=0.3 \mathrm{c}$ and $\Delta \phi \stackrel{\text { prec }}{=} 2^{\circ}$. However, the full multibeam structure may reach tens of degrees.

To reach agreement of the model with the observed "superluminal" velocities of VLBI-components it is necessary to suggest a comet-like shape (a head-tail structure) for the circum-nuclear gas clouds. In fact, this shape seems quite natural if one remembers the strong gas flows observed in active nuclei in the form of hot supersonic winds. 
Taking into account that clouds may be elongated in radial direction near active nuclei one obtains $V_{\text {obs }}=V_{\text {prec }} \operatorname{Cos} \psi / \operatorname{Sin} \beta$, where $\beta$ is the angle of the tail deviation from the pure radial direction, and $\psi$ the angle between the tail and the picture plane. From the formula one may reproduce the observed value $\mathrm{V}_{\text {obs }}>3 \mathrm{c}$ if $\operatorname{Cos} \psi / \operatorname{Sin} \beta>10$.

The following conclusions from the "screen" model must be tested by future observations:

1. "Superluminal" motions should be observed not only at radio frequencies, but also in another range, in particular in the optical one, where the emission must precede the radio emission by months or years.

2. "Superluminal" motions must be observed only in very variable sources (although an affirmation to the contrary does not invalidate the model).

3. Variable emission with cycle times longer than one year must not arise in the nuclei, but in areas of interactions located at distances of tens of parsecs from the nucleus.

4. "Superluminal" velocities must increase the further one gets from a nucleus for the expansion case, and decrease the closer one gets to the nucleus for the contraction case.

5. Together with "superluminal" motions of VLBI-components one may expect a still more rapid removal of the edges of hot radio-spots observed in the extended components of radio sources of type FR II.

6. Comet-like gas structures must be observed as elongated filaments in the forbidden line region. 